\title{
Planning and Project of Medium Voltage Electric Power Distribution Systems
}

\author{
V. C. Cunha and J. R. S. Mantovani, Member, IEEE
}

\begin{abstract}
This paper presents a technique to solve the problem of planning and project of medium voltage distribution systems. The problem is modeled as a mixed integer nonlinear programming model. The solution technique proposed to solve this model is based on a tabu search algorithm which is capable of handling large-scale nonlinear problems. Numerical results from a 50-bus test system show the effectiveness of the proposed approach.
\end{abstract}

Keywords - Medium Voltage Distribution Systems, Planning, Project, Tabu Search.

\section{NOMENCLATURA}

\section{A. Conjuntos}

$N R$

$N S$

Número de ramos do sistema;

$N P$

Número de subestações do sistema;

Número de postes alocados no sistema;

NE Número de estruturas existentes no sistema;

$N B$

Número de barras do sistema;

\section{B. Parâmetros}

$C S_{i}$

Custo de construção e/ou repotencialização da subestação $i$ (US\$);

\section{$\mathrm{CFC}_{i}$}

$C_{k W h} \quad$ Custo do kWh (US\$/(MVA*h*km) Custo dos cabos instalados no ramo $i$

$T$

$S_{i}$

$d_{i}$

$C P_{i}$

$C E_{i}$

$C E S$

$E S_{j}$

$V_{n}$

$\Delta V_{-}$

$\Delta V_{+}$

$V_{f}$

Número de horas totais no período de planejamento;

Perdas ôhmicas no cabo $i$ (MVA);

Comprimento do cabo $i(\mathrm{~km})$;

Custo de aquisição do poste do tipo $i$;

Custo da estrutura de sustentação do tipo

$$
i
$$

Custo de espaçadores (rede compacta);

Número de espaçadores presentes no ramo $j$;

Tensão nominal;

Máxima variação negativa da tensão;

Máxima variação positiva da tensão;

Tensão de alimentação em cada barra;

V. C. Cunha, Universidade Estadual Paulista (UNESP), Ilha Solteira, São Paulo, Brasil, viniciuscarnelossi@hotmail.com.

J. R. S. Mantovani, Universidade Estadual Paulista (UNESP), Ilha Solteira, São Paulo, Brasil, mant@dee.feis.unesp.br.

Projeto de pesquisa financiado pelo CNPq (Processos 506658/2010-4 e 305371/2012-6).
Caputi $_{i} \quad$ Capacidade utilizada da subestação $i$;

CapMax $_{i} \quad$ Capacidade máxima da subestação $i$;

$P_{i} \quad$ Potência ativa líquida injetada na barra $i$;

$Q_{i} \quad$ Potência reativa líquida injetada na barra

$P_{G i} \quad$ Potência ativa gerada na barra $i$;

$Q_{G i} \quad$ Potência reativa gerada na barra $i$;

$P_{c i} \quad$ Potência ativa consumida na barra $i$;

$Q_{c i} \quad$ Potência reativa consumida na barra $i$;

$t \quad$ Tap dos reguladores de tensão;

$\theta$

$V \quad$ Magnitude de tensões nas barras.

Capmax $_{\text {cabi }}$ Capacidade máxima do cabo $i$;

$Z_{\text {cabi }} \quad$ Impedância por quilômetro do cabo $i$ $(\Omega / \mathrm{km})$;

$d_{i-j} \quad$ Distância entre as barras $i$ e $j$;

$I_{i-j}$

$H_{i}$

$L_{i}$

$E_{i}$

$F_{R}$

$F_{p}$

$F_{s}$

$H_{s}$

Corrente que passa pelo condutor entre as barras $i$ e $j$;

Altura útil do poste $i$;

Altura nominal do poste $i$;

Altura de engastamento do poste $i$;

Força resultante;

Força de tração nos cabos da rede primária;

Força de tração dos cabos na rede secundária;

Altura média de instalação do circuito da rede secundária;

Força de tração dos cabos da rede telefônica;

Altura média de instalação da rede telefônica.

Força aplicada ao poste $j$ referente ao eixo $\mathrm{x}$;

Força aplicada ao poste $j$ referente ao eixo y;

Força resultante no condutor $i$ aplicada ao poste j;

Ângulo de incidência do condutor $i$ no poste $j$, referente ao eixo normal;

Esforço para o qual o poste deve ser $F_{\text {jtotal } \quad \text { projetado. }}$ 


\section{Variáveis}

$X S_{i}$

Variável binária que define a existência da subestação $i$;

$X C_{i}$

Variável binária que define a existência do cabo $i$;

$X P_{i}$ Variável binária que define a existência do poste $i$;

$X E_{i}$ Variável binária que define a existência da estrutura de sustentação $i$; Variável binária que define a existência de espaçadores no ramo $j$.

\section{INTRODUÇÃO}

$\mathrm{N}_{\mathrm{g}}^{\mathrm{o}}$ O planejamento e projetos de sistemas é essencial garantir que o crescimento da demanda por eletricidade, devido aos aumentos da taxa de crescimento de consumo e a alta da densidade de carga, seja acompanhado pela adição de novos elementos de rede nos sistemas de distribuição, os quais devem ser técnica e economicamente viáveis. No futuro, mais do que no passado, empresas distribuidoras necessitam de uma ferramenta computacional rápida e eficiente para a análise das consequências de diferentes alternativas de planejamento propostas e, consequentemente, avaliarem os seus respectivos impactos no sistema para suprir os consumidores com a energia elétrica necessária, econômica, confiável e segura [1].

O crescimento contínuo do custo da energia, equipamentos e serviços, exige o desenvolvimento e o uso de avançados sistemas de planejamento e projetos de sistemas de distribuição através de técnicas inteligentes pelas empresas distribuidoras. O sistema de distribuição é particularmente importante para o cenário elétrico global por dois principais motivos: (i) é o sistema que está mais próximo do consumidor, e; (ii) possui altos custos de investimentos. Como este sistema é o mais próximo dos consumidores, falhas neste sistema têm probabilidade de afetá-los mais diretamente do que, por exemplo, falhas na transmissão e na geração, que podem eventualmente não gerar interrupções [1].

O problema de planejamento e projetos de sistemas de distribuição pode ser formulado considerando-se um novo sistema que deve ser construído para atender à demanda de um grupo de consumidores ou um sistema genérico que atenda a um número fixo de consumidores, porém há crescimento temporal e espacial da demanda, resultando na necessidade de expansão da rede. Portanto, deve-se considerar a construção de novas linhas e/ou troca das linhas existentes por outras de maior capacidade, construção de subestações e ampliação das existentes e alocação de postes, estruturas mecânicas de sustentação, de suspensão e de equipamentos. Os custos relacionados a estes investimentos e de operação, mais especificamente perdas técnicas, satisfazendo um conjunto de restrições operacionais, físicas e financeiras, constituem o problema de planejamento e projetos de redes [2].

As principais dificuldades na resolução deste problema são a natureza combinatória do modelo no problema de planejamento que apresenta um espaço de solução de grande dimensão, estrutura multimodal com um número elevado de ótimos locais e variáveis de decisão inteiras. Na literatura, encontram-se diferentes propostas de trabalhos que tratam tanto do modelo de planejamento de sistemas de energia elétrica como das técnicas de solução. As técnicas propostas para solução do problema de planejamento ótimo de sistemas de distribuição são de otimização clássica (Branch-andBound, Decomposição de Benders e modelos de transportes) [3] e [4]; métodos heurísticos [5] e as meta-heurísticas (algoritmos genéticos [6] e [7]; evolução computacional [8]; busca tabu [9] e sistema de colônia de formigas [10]). Em [11] e [12], são propostos modelos multiobjetivos para o planejamento de redes de distribuição de média tensão. Em [13], uma metodologia, baseada em um modelo de otimização multiobjetivo dinâmico para o planejamento simultâneo da expansão de redes de distribuição e da confiabilidade, é proposta. No modelo de planejamento da expansão da rede são considerados a construção de novas subestações e repotencialização das subestações existentes, construção de novos ramais e circuitos e recondutoramento de circuitos existentes. O projeto de confiabilidade da rede consiste na alocação de chaves de manobras e ramais de interconexões, com vista à restauração da rede em condições de contingências, usando o critério $n-1$, e o conceito de energia não distribuída para avaliar a confiabilidade da rede. A metaheurística busca tabu multiobjetivo é utilizada para resolver o problema de otimização. Em [14], o problema de planejamento de redes de distribuição é formulado como um modelo de programação não linear inteiro misto estático, em que se consideram as ações de planejamento da rede e a melhoria da confiabilidade do sistema operando no estado restaurativo através da alocação de chaves de manobras. O problema é resolvido de forma heurística em que o problema de planejamento é resolvido através de um algoritmo de busca tabu multiobjetivo e o problema de alocação de chaves de manobras através de um algoritmo genético simples, ou seja, para cada topologia da rede obtida através do critério de vizinhança da busca tabu se efetua a alocação otimizada de chaves de manobras usando o algoritmo genético, cuja função objetivo considerada é o custo da energia não distribuída. Em [15], formula-se o problema de planejamento de redes de distribuição como um modelo estático de programação não linear inteira mista em que se consideram as ações de planejamento básicas e a minimização dos custos de operação e investimentos. A técnica de solução adotada para o problema é um algoritmo de busca dispersa, consistindo a mesma na principal contribuição deste trabalho.

Os trabalhos encontrados na literatura consideram apenas o planejamento de médio e longo prazo dos sistemas de distribuição não modelando a especificação dos componentes de sustentação e construção da rede, tais como as estruturas mecânicas necessárias e especificações dos postes. Neste trabalho, propõe-se um modelo de programação não linear inteiro misto (PNLIM) que contempla tanto o planejamento estático da rede de distribuição de média tensão como o seu projeto, em que devem ser especificados de forma otimizada, através de equações que fazem parte das restrições do problema e da função objetivo, os cálculos do projeto mecânico dos elementos de sustentação da rede. A solução proposta para este problema é dada através de técnicas heurísticas e da meta-heurística busca tabu (BT) para encontrar uma solução otimizada de boa qualidade que tenha os custos 
de instalações e operação minimizados, atendendo a todos os critérios de qualidade e confiabilidade impostos aos sistemas de distribuição de energia. A solução encontrada deve fornecer as ações que devem ser realizadas tanto nas etapas de planejamento do sistema elétrico quanto na de projetos das estruturas mecânicas, tornando-as possíveis de serem implementadas na prática pelas empresas que fornecem este serviço.

Este trabalho possui cinco seções. Na seção II, o problema de planejamento e projetos de sistema de distribuição de energia elétrica é formulado matematicamente. Na seção III, aborda-se a técnica de $B T$ empregada, em que cada etapa do processo de otimização é detalhada. As seções IV e V apresentam, respectivamente, os resultados e as conclusões da utilização deste método de planejamento para um sistema teste com 50 barras.

\section{O PROBLEMA DE PLANEJAMENTO E PROJETOS DE REDES DE DISTRIBUIÇÃO}

O problema de planejamento e projetos de sistemas de distribuição de energia elétrica é modelado como um problema de programação não linear inteira mista (PNLIM) [2]. Desta forma, propõe-se a minimização dos custos referentes ao planejamento do sistema elétrico, que corresponde à alocação de novos circuitos ou recondutoramento de circuitos já existentes, à construção de novas subestações ou repotencialização de subestações já existentes e às perdas técnicas na linha. Adicionalmente a estas ações de planejamento comumente encontradas na literatura, neste trabalho propõe-se a minimização destes custos considerando o projeto mecânico da rede, que corresponde à alocação de estruturas de suspensão (postes) e sustentação para os circuitos e a alocação de espaçadores entre as fases.

A minimização destes custos está sujeita a atender às restrições impostas para operação dos sistemas de distribuição, como a radialidade do sistema, capacidade máxima dos alimentadores e subestações, esforços de tração nas estruturas mecânicas, atendimento da demanda máxima das cargas e o limite da magnitude da tensão nas barras, garantindo a qualidade do fornecimento do serviço [1].

\section{Formulação matemática}

No desenvolvimento do modelo matemático do problema, consideram-se duas funções objetivos, em que a primeira, $F O_{1}$, representa os custos fixos e variáveis do planejamento do sistema elétrico (1), e a segunda, $\mathrm{FO}_{2}$, representa os custos das estruturas de sustentação e suspensão da rede (2).

$$
\begin{aligned}
& F O_{1}=\sum_{i=1}^{N S} C S_{i} X S_{i}+\sum_{i=1}^{N R} C F C_{i} X C_{i} d_{i}+\sum_{i=1}^{N R} C_{k W h} X C_{i} T\left|S_{i}\right| d_{i} \\
& F O_{2}=\sum_{i=1}^{N P} C P_{i} X P_{i}+\sum_{i=1}^{N E} C E_{i} X E_{i}+\sum_{j=1}^{N R} C E S . X E S_{j} . E S_{j}
\end{aligned}
$$

Na equação (1), o primeiro termo é o custo da subestação $i$, o segundo termo é o custo de alocação do cabo no caminho $i$, considerando a bitola e o comprimento do condutor e o último termo é o custo das perdas ôhmicas no alimentador $i$, no período de planejamento.

$\mathrm{Na}$ equação (2), o primeiro termo é o custo de instalação dos postes de acordo com as exigências do projeto e normas das empresas, o segundo termo é o custo de instalação das estruturas de sustentação da rede e o último termo é o custo dos espaçadores que devem ser instalados nos alimentadores para sustentação mecânica, considerando-se a utilização de redes compactas.

As equações de (3) a (7) são as restrições impostas que garantem a qualidade do fornecimento de energia, o atendimento da demanda dos consumidores e a confiabilidade do sistema.

$V_{n \cdot}\left(1+\Delta V_{-}\right) \leq V_{f} \leq V_{n} \cdot\left(1+\Delta V_{+}\right)$

$P_{G i}-P_{C i}-P_{i}(\theta, V, t)=0 \quad i=1, \ldots, N B$

$Q_{G i}-Q_{C i}-Q_{i}(\theta, V, t)=0 \quad i=1, \ldots, N B$

$\operatorname{Capmax}_{c a b i}=\left|\left(Z_{c a b i} \cdot d_{i-j} \cdot\left|I_{i-j}\right|^{2}\right)\right|$

Caputi $_{i} \leq$ Capmax $_{i}$

A equação (3) modela a variação da magnitude da tensão nas barras. Esta variação é limitada em $\pm \% \Delta \mathrm{V}$ da tensão nominal, conforme a literatura [16]. As equações (4) e (5) representam as equações estáticas de fluxo de potência e visam ao atendimento das demandas de potência ativa e reativa nos nós de consumo. A equação (6) define a máxima capacidade de transferência de potência que o condutor $i$ pode comportar e a equação (7) define a potência máxima que o transformador da subestação $i$ pode fornecer.

As equações de (8) a (13) representam os cálculos necessários do projeto mecânico da rede, que são os esforços nos quais as estruturas de suspensão e de sustentação mecânicas estão sujeitas, considerando o método de transferência de esforços a $20 \mathrm{~cm}$ do topo de cada poste [17].

$E_{i}=0,10 \cdot L_{i}+0,6$

$H_{i}=L_{i}-E_{i}-0,2$

$F_{x j}=F_{r i} \cdot \cos \left(\alpha_{i}\right)+\ldots+F_{r k} \cdot \cos \left(\alpha_{k}\right)$

$F_{y j}=F_{r i} \cdot \operatorname{sen}\left(\alpha_{i}\right)+\ldots+F_{r k} \cdot \operatorname{sen}\left(\alpha_{k}\right)$

$F_{j t o t a l}=\sqrt{F_{x j}^{2}+F_{y j}^{2}}$

$F_{R}=F_{p}+\left(\frac{F_{s} \cdot H_{s}}{H_{i}}\right)+\left(\frac{F_{t} \cdot H_{t}}{H_{i}}\right)$

Os postes são padronizados por normas nas alturas de 9, 11 ou 12 metros, de acordo com as suas aplicações [17]. Os postes de 9 metros são utilizados em condições que existem apenas a rede secundária e telecomunicação. Já os postes de 11 metros são utilizados para condições que existem as redes primárias, secundárias e telecomunicação. Os postes de 12 metros são utilizados em postos de transformação. Definida a 
altura do poste, define-se também o engastamento deste, que é o comprimento de sua fixação ao solo, pelas equações (8) e (9).

As equações (10) e (11) definem o cálculo dos esforços mecânicos projetados nos eixos x e y, respectivamente, sendo que a equação (12) define o esforço resultante a que o poste está submetido. A equação (13) expressa o somatório dos esforços mecânicos no poste devido aos cabos das redes primária, secundária e de telecomunicação, representados pelos primeiro, segundo e terceiro termos, respectivamente. $\mathrm{O}$ segundo e terceiro termos são deslocados para o ponto a 20 $\mathrm{cm}$ do topo do poste, proposto pela técnica de cálculo dos esforços [17].

Para solução deste problema de otimização matemática propõe-se um algoritmo de Busca Tabu (BT) dedicado [18], que é amplamente empregado para solução deste tipo de problema. Este algoritmo é uma ferramenta robusta para resolver problemas de PNLIM e pode obter boas soluções com baixo tempo de processamento para o problema de planejamento e projetos de sistemas de distribuição de energia elétrica de média tensão.

\section{TÉCNICA DE SOLUÇÃO}

$O$ algoritmo de $B T$ visa à resolução de problemas de otimização partindo de uma configuração inicial em que, a partir desta solução, define-se o conjunto de soluções vizinhas. Soluções vizinhas são todas as soluções que apresentam atributos semelhantes à solução que as originou. Após a obtenção do subconjunto de soluções vizinhas factíveis, é necessário avaliá-las utilizando a função de adaptação $(F A)$ e aplicar o critério de aspiração. Determinando a melhor solução do conjunto da vizinhança, armazenam-se os seus atributos na Lista Tabu $(L T)$.

A $L T$ tem como finalidade eliminar a ciclagem no espaço de busca, evitando que o algoritmo se limite a um espaço local ótimo não atingindo a região ótima global do problema em que se encontra a solução ótima desejada. Para isso, a $L T$ armazena atributos específicos das melhores soluções e compara com os atributos da solução corrente em análise. Caso a solução corrente não tenha atributos proibidos ou satisfaça o critério de aspiração, ela é escolhida. Do contrário, bloqueia esta solução e se escolhe a melhor solução não bloqueada pela $L T$. Após a análise da $L T$ e do critério de aspiração, tem-se a melhor solução encontrada para a iteração que está sendo analisada. Esta solução passa pelo processo de intensificação [18], a fim de minimizar o valor das $F O$ s do problema e, consequentemente, da $F A$.

Na Fig. 1 ilustra-se o diagrama de blocos do algoritmo de $B T$ para o problema de planejamento e projetos de sistemas de distribuição de energia elétrica. Neste diagrama, encontram-se ilustradas as seguintes etapas do algoritmo $B T$ : Determinação da configuração inicial; Determinação das soluções vizinhas; Cálculos do fluxo de potência, Cálculos mecânicos e da $F A$; $L T$ e critério de aspiração; Intensificação e critério de parada.

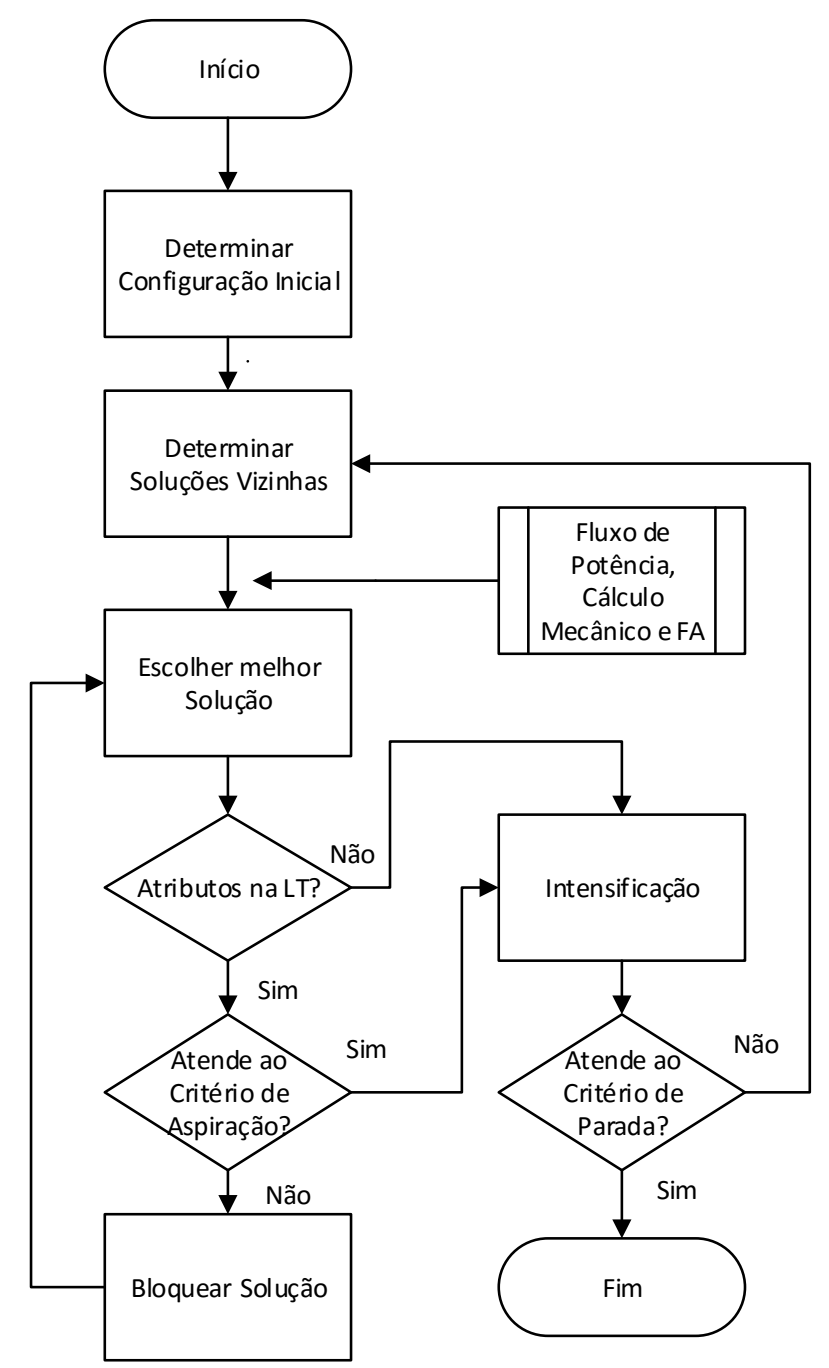

Figura 1. Diagrama de blocos do algoritmo de BT.

\section{E. Codificação do problema}

A codificação do problema utilizada neste trabalho é exemplificada através do sistema básico ilustrado na Fig. 2, que possui 2 subestações existentes (S1 e S2) e uma possível subestação para construção (S3), 3 alimentadores existentes $\{1,2,9\}$ e 7 possíveis alimentadores para construção $\{3,4,5$, $6,7,8,10\}$.

Analisando a Fig. 2, verifica-se que existem dezenas de soluções de construção de circuitos e subestações para o atendimento das barras de demanda pelas subestações. Para codificar as possíveis soluções de planejamento e projeto para este sistema é necessário definir um vetor que armazenará um valor inteiro de acordo com o modelo do cabo adotado, e.g. " 2 " ou " 5 ", caso esteja conectado ou " 0 " caso este esteja desconectado.

Por exemplo, a Fig. 3 ilustra o vetor de conexão do alimentador para uma solução do sistema da Fig. 2. 

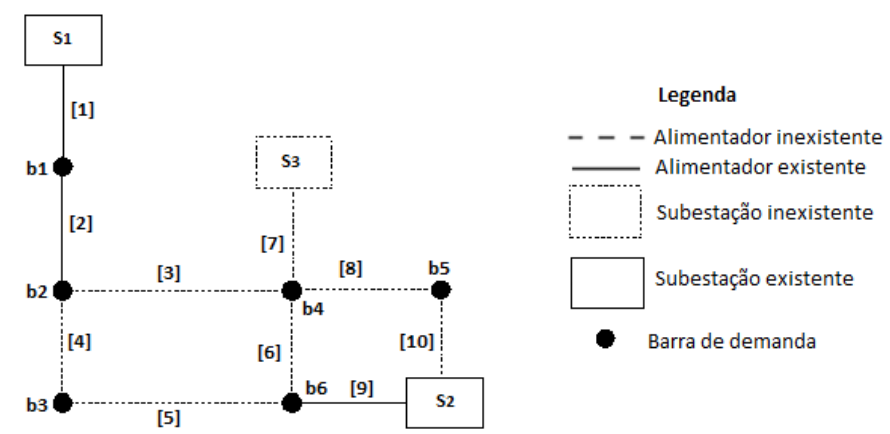

Figura 2. Exemplo fictício de rede elétrica malhada.

\begin{tabular}{|l|l|l|l|l|l|l|l|l|l|}
1 & 2 & 3 & 4 & 5 & 6 & 7 & 8 & 9 & 10 \\
\hline 2 & 1 & 0 & 0 & 3 & 1 & 0 & 0 & 2 & 2 \\
\hline
\end{tabular}

Figura 3. Exemplo de vetor para codificação do problema.

A partir do vetor dado na Fig. 3, representa-se a topologia proposta para esta solução para o sistema através da Fig. 4.

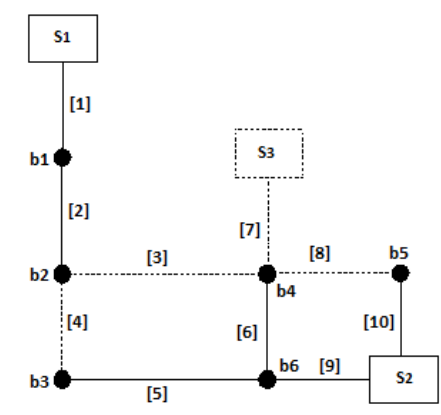

Figura 4. Topologia para a solução do exemplo.

Após a estruturação do problema, pode-se avaliar o atendimento das restrições impostas por meio da análise dos parâmetros elétricos do sistema. Para isso, utilizou-se o algoritmo de fluxo de potência proposto na literatura [19].

O algoritmo proposto para solução do problema é composto pelos seguintes passos:

i. Geração da configuração inicial. Este procedimento consiste em determinar uma configuração inicial factível para o problema. Esta configuração inicial é parte importante do algoritmo, pois influencia diretamente no esforço computacional que a $B T$ será submetida durante o processo iterativo [18]. Portanto, a utilização de uma estratégia para gerar a configuração inicial de boa qualidade permite com que o algoritmo obtenha soluções ótimas ou quase ótimas com menor esforço computacional. A heurística construtiva $(H C)$, adotada neste trabalho para gerar a configuração inicial, consiste em alimentar as barras por subestações existentes até que se conectem todas as barras do sistema ou atinjam os limites operacionais das subestações. No segundo caso, considera-se a inclusão de novas subestações ou a repotencialização das antigas. $\mathrm{O}$ critério de parada da $H C$ é atingido quando todas as barras do sistema forem alimentadas. Esta heurística garante a radialidade do sistema, tornando a configuração inicial factível sob o aspecto da radialidade. ii. Determinação das Soluções Vizinhas. O critério proposto para a escolha da vizinhança é inspirado na técnica de troca de ramos proposta por Goswami [5], no qual se insere uma nova linha no sistema, tornando-o não radial e, em seguida, elimina-se uma linha existente para que o sistema retome sua característica radial. Repete-se este processo até o esgotamento de todas as possibilidades, obtendo-se assim o subconjunto de soluções vizinhas para cada solução sob análise.

iii. Cálculos do fluxo de potência, cálculos mecânicos e função de adaptação. O fluxo de potência é calculado para todas as configurações vizinhas determinadas no subconjunto da etapa $i i$ utilizando o algoritmo proposto em [19]. Através do algoritmo de fluxo de potência é possível conhecer as características elétricas do sistema para cada solução vizinha, para, posteriormente, avaliá-las tanto elétrica quanto mecanicamente. O Cálculo mecânico das estruturas consiste em utilizar as equações de (8) a (13) para determinar os esforços em que as estruturas de suspensão e sustentação estarão submetidas para cada solução analisada. Para isto, utiliza-se o método da transferência de esforços para $20 \mathrm{~cm}$ do topo, conforme definido em [17]. Determina-se também o tipo de estrutura de sustentação mais adequado para cada barra do sistema em estudo, conforme apresentado em [20]. Definidos os cálculos do fluxo de potência e do cálculo mecânico para cada solução vizinha é possível avaliar as restrições do modelo e determinar a função de adaptação $(F A)$, que é formulada em (14), para cada solução.

iv. Função de Adaptação: A função de adaptação é dada pelo somatório dos termos das funções objetivos do problema e das penalizações impostas à solução por não cumprimento das restrições. Portanto, a solução ótima para a $F A$ é dada pela minimização do custo dado pelo somatório das funções objetivos sujeita ao cumprimento das restrições do problema, já que qualquer violação resultará em uma penalização na $F O$ que tornará a configuração sob análise pouco atrativa.

$F A=F O_{1}+F O_{2}+P E N$

v. Lista Tabu e critério de aspiração. Definida a FA para cada solução vizinha, escolhe-se a solução que obtiver o menor valor de $F A$, ou seja, aquela que representa a melhor solução do subconjunto de soluções vizinhas. Esta solução tem seus atributos analisados, em que caso estejam proibidos e a $F A$ não atender ao critério de aspiração, esta solução será bloqueada e deve-se escolher a nova melhor solução não proibida para o problema. Os atributos armazenados na lista tabu são os ramos de conexões da melhor solução encontrada pela BT que são diferentes da topologia inicial em cada iteração. Repete-se este passo até que a solução analisada não tenha atributos proibidos na $L T$ ou ela atenda ao critério de aspiração. $\mathrm{O}$ critério de aspiração adotado é que a configuração corrente em análise deve apresentar $F A$ de menor valor do que todas as soluções já investigadas.

vi. Intensificação. $\mathrm{O}$ processo de intensificação é realizado após a análise da $L T$ e do critério de aspiração. Este processo consiste na variação da bitola dos condutores 
que interligam as barras do sistema visando à redução da $F A$ e, consequentemente, da $F O$. Caso a modificação realizada resulte em uma redução da $F A$ esta modificação torna-se permanente. Do contrário, desfaz-se desta alteração.

vii. Critério de Parada. Após o processo de intensificação, tem-se determinada a melhor solução não proibida para o subconjunto de vizinhos estudados. Caso o critério de parada, determinado por um número fixo de iterações ou a não modificação da $F A$, seja atingido, o algoritmo é finalizado. Caso contrário, a melhor solução desta iteração será utilizada como configuração inicial para a próxima iteração.

\section{RESULTADOS E DISCUSSÕES}

Os testes e simulações foram realizados usando um computador com processador AMD Turion ${ }^{\mathrm{TM}} \mathrm{X} 2$ Dual-Core Mobile RM-70 2.0 GHz e 3GB de memória RAM. Os testes visam à demonstração da eficiência e a aplicação da metodologia proposta. A programação do algoritmo foi feita na linguagem de programação $\mathrm{C}^{++}$. Para os resultados discutidos, a $F A$ é a própria $F O$ geral do problema, pois, para o sistema estudado, as melhores soluções atendem a todas as restrições impostas no modelo, eliminando assim o terceiro item de penalização (PEN) da equação (14).

\section{F. Parâmetros e Dados Utilizados no Sistema Teste}

Nesta seção, apresentam-se os parâmetros e dados utilizados na simulação do sistema teste. Na tabela I, definemse os tipos (bitolas) de cabos utilizados.

TABELA I

MODELOS DE CABOS DO SISTEMA

\begin{tabular}{c|c|c|c|c|c}
\hline $\begin{array}{c}\text { Tipo } \\
\text { de } \\
\text { cabo }\end{array}$ & $\begin{array}{c}\text { Smax } \\
\text { (MVA) }\end{array}$ & $\begin{array}{c}\mathbf{R} \\
(\mathbf{p u} / \mathbf{K m})\end{array}$ & $\begin{array}{c}\mathbf{X} \\
(\mathbf{p u} / \mathbf{K m})\end{array}$ & $\begin{array}{c}\text { Custo } \\
\text { (US\$/Km) }\end{array}$ & $\begin{array}{c}\text { Bitola } \\
\left.\mathbf{( m m}^{2}\right)\end{array}$ \\
\hline $\mathbf{1}$ & 9 & 0,3655 & 0,2520 & 20 & 25 \\
\hline $\mathbf{2}$ & 11 & 0,2921 & 0,2466 & 30 & 35 \\
\hline $\mathbf{3}$ & 12,5 & 0,2359 & 0,2402 & 40 & 50 \\
\hline $\mathbf{4}$ & 14 & 0,1932 & 0,2279 & 50 & 70 \\
\hline $\mathbf{5}$ & 19 & 0,1827 & 0,1260 & 60 & 90 \\
\hline $\mathbf{6}$ & 22,4 & 0,146 & 0,1233 & 70 & 120 \\
\hline $\mathbf{7}$ & 25 & 0,118 & 0,1201 & 80 & 150 \\
\hline $\mathbf{8}$ & 28 & 0,0966 & 0,1140 & 90 & 185 \\
\hline
\end{tabular}

$\mathrm{Na}$ tabela II, definem-se as características topológicas dos ramos do sistema.

TABELA II CARACTERÍSTICAS DOS RAMOS DO SISTEMA

\begin{tabular}{c|c|c|c|c|c|c|c}
\hline Ramo & nó $_{\mathbf{i}}$ & $\mathbf{n o ́}_{\mathbf{j}}$ & $\begin{array}{c}\boldsymbol{d i} \boldsymbol{i}-\boldsymbol{j} \\
(\mathbf{k m})\end{array}$ & $\mathbf{R a m o}$ & $\mathbf{n o ́}_{\mathbf{i}}$ & $\mathbf{n o ́}_{\mathbf{j}}$ & $\begin{array}{c}\boldsymbol{d i} \boldsymbol{i} \boldsymbol{j} \\
(\mathbf{k m})\end{array}$ \\
\hline $\mathbf{4}$ & 101 & 1 & 2,81 & $\mathbf{3 5}$ & 6 & 28 & 5 \\
\hline $\mathbf{5}$ & 101 & 3 & 2,18 & $\mathbf{3 6}$ & 104 & 30 & 2,81 \\
\hline $\mathbf{6}$ & 3 & 4 & 3,12 & $\mathbf{3 7}$ & 30 & 29 & 3,12 \\
\hline $\mathbf{7}$ & 4 & 7 & 2,5 & $\mathbf{3 8}$ & 30 & 43 & 4,06 \\
\hline $\mathbf{8}$ & 4 & 5 & 3,12 & $\mathbf{3 9}$ & 43 & 37 & 2,5 \\
\hline
\end{tabular}

\begin{tabular}{|c|c|c|c|c|c|c|c|}
\hline 9 & 7 & 8 & 3,12 & 40 & 37 & 31 & 1,87 \\
\hline 10 & 5 & 6 & 2,5 & 41 & 31 & 10 & 3,12 \\
\hline 11 & 1 & 9 & 3,43 & 42 & 13 & 43 & 3,75 \\
\hline 12 & 1 & 2 & 3,12 & 43 & 12 & 45 & 2,5 \\
\hline 13 & 9 & 10 & 7,18 & 44 & 45 & 44 & 2,18 \\
\hline 14 & 102 & 14 & 3,75 & 45 & 44 & 38 & 3,12 \\
\hline 15 & 14 & 15 & 3,75 & 46 & 38 & 39 & 3,43 \\
\hline 16 & 15 & 16 & 2,81 & 47 & 39 & 32 & 4,06 \\
\hline 17 & 102 & 11 & 2,81 & 48 & 39 & 33 & 2,81 \\
\hline 18 & 11 & 12 & 3,12 & 49 & 33 & 8 & 4,68 \\
\hline 19 & 12 & 13 & 4,37 & 50 & 33 & 34 & 1,87 \\
\hline 20 & 19 & 20 & 3,12 & 51 & 34 & 35 & 2,18 \\
\hline 21 & 18 & 19 & 2,5 & 52 & 35 & 36 & 2,18 \\
\hline 22 & 17 & 18 & 4,06 & 53 & 103 & 36 & 2,5 \\
\hline 23 & 9 & 17 & 4,3 & 54 & 103 & 28 & 3,12 \\
\hline 24 & 18 & 21 & 3,12 & 55 & 103 & 41 & 3,12 \\
\hline 25 & 104 & 21 & 2,5 & 56 & 41 & 40 & 2,5 \\
\hline 26 & 104 & 22 & 3,75 & 57 & 40 & 16 & 2,5 \\
\hline 27 & 9 & 22 & 4,68 & 58 & 41 & 42 & 3,75 \\
\hline 28 & 22 & 23 & 3,43 & 59 & 42 & 48 & 2,5 \\
\hline 29 & 23 & 24 & 2,81 & 60 & 48 & 49 & 3,75 \\
\hline 30 & 24 & 25 & 2,18 & 61 & 49 & 50 & 2,18 \\
\hline 31 & 25 & 8 & 2,81 & 62 & 42 & 47 & 3,12 \\
\hline 32 & 8 & 27 & 3,75 & 63 & 47 & 46 & 3,12 \\
\hline 33 & 27 & 26 & 3,43 & 64 & 46 & 14 & 3,43 \\
\hline 34 & 27 & 28 & 3,12 & & & & \\
\hline
\end{tabular}

Na tabela III, apresentam-se as subestações existentes no sistema, assim como as respectivas capacidades de demanda de seus transformadores.

TABELA III SUBESTAÇÕES DO SISTEMA

\begin{tabular}{c|c|c|c}
\hline \multirow{3}{*}{ Existente } & Subestação & $\begin{array}{c}\text { Capacidade } \\
\text { (MVA) }\end{array}$ & Custo Fixo (US\$) \\
\cline { 2 - 4 } & 101 & 33,4 & 100 \\
\hline \multirow{3}{*}{ Futuras } & 102 & 30 & 80 \\
\cline { 2 - 4 } & 103 & 22 & 200 \\
\hline
\end{tabular}

Na tabela IV, apresentam-se as demandas de cada barra do sistema. Na solução do fluxo de potência, usando modelo de carga de potência constante, adotou-se fator de potência 0,92 para todas as cargas.

TABELA IV

DEMANDA ESTÁTICA DAS BARRAS DO SISTEMA

\begin{tabular}{c|c|c|c|c|c}
\hline Barra & $\begin{array}{c}\text { Demanda } \\
\text { (MVA) }\end{array}$ & Barra & $\begin{array}{c}\text { Demanda } \\
\text { (MVA) }\end{array}$ & Barra & $\begin{array}{c}\text { Demanda } \\
\text { (MVA) }\end{array}$ \\
\hline $\mathbf{1}$ & 3,3 & $\mathbf{1 8}$ & 0,9 & $\mathbf{3 5}$ & 0 \\
\hline
\end{tabular}




\begin{tabular}{c|c|c|c|c|c}
\hline $\mathbf{2}$ & 1,1 & $\mathbf{1 9}$ & 1 & $\mathbf{3 6}$ & 0,2 \\
\hline $\mathbf{3}$ & 0,4 & $\mathbf{2 0}$ & 0,5 & $\mathbf{3 7}$ & 1 \\
\hline $\mathbf{4}$ & 1,4 & $\mathbf{2 1}$ & 0,5 & $\mathbf{3 8}$ & 0 \\
\hline $\mathbf{5}$ & 2 & $\mathbf{2 2}$ & 0,5 & $\mathbf{3 9}$ & 0,8 \\
\hline $\mathbf{6}$ & 0,6 & $\mathbf{2 3}$ & 0,5 & $\mathbf{4 0}$ & 1 \\
\hline $\mathbf{7}$ & 0,2 & $\mathbf{2 4}$ & 0,5 & $\mathbf{4 1}$ & 0,3 \\
\hline $\mathbf{8}$ & 1,5 & $\mathbf{2 5}$ & 0,6 & $\mathbf{4 2}$ & 0 \\
\hline $\mathbf{9}$ & 1,9 & $\mathbf{2 6}$ & 0 & $\mathbf{4 3}$ & 0 \\
\hline $\mathbf{1 0}$ & 2 & $\mathbf{2 7}$ & 0 & $\mathbf{4 4}$ & 0,5 \\
\hline $\mathbf{1 1}$ & 0,2 & $\mathbf{2 8}$ & 0,4 & $\mathbf{4 5}$ & 0,5 \\
\hline $\mathbf{1 2}$ & 1 & $\mathbf{2 9}$ & 0,6 & $\mathbf{4 6}$ & 0 \\
\hline $\mathbf{1 3}$ & 0,9 & $\mathbf{3 0}$ & 2 & $\mathbf{4 7}$ & 0,5 \\
\hline $\mathbf{1 4}$ & 0,8 & $\mathbf{3 1}$ & 0,6 & $\mathbf{4 8}$ & 0,5 \\
\hline $\mathbf{1 5}$ & 1 & $\mathbf{3 2}$ & 0 & $\mathbf{4 9}$ & 0 \\
\hline $\mathbf{1 6}$ & 1,3 & $\mathbf{3 3}$ & 1,8 & $\mathbf{5 0}$ & 0 \\
\hline $\mathbf{1 7}$ & 0,5 & $\mathbf{3 4}$ & 0,9 & & \\
\hline & & & & & \\
\hline
\end{tabular}

$\mathrm{Na}$ tabela $\mathrm{V}$, encontram-se as características gerais dos postes utilizados neste trabalho.

TABELA $\mathrm{V}$

\begin{tabular}{c|c|c}
\hline $\begin{array}{c}\text { CARACTERÍSTICAS GERAIS DOS POSTES } \\
\begin{array}{c}\text { Comprimento } \\
(\mathbf{m})\end{array}\end{array}$ & $\begin{array}{c}\text { Capacidade } \\
\text { (daN) }\end{array}$ & $\begin{array}{c}\text { Custo } \\
\text { (US\$) }\end{array}$ \\
\hline $\mathbf{9}$ & 200 & 118,69 \\
\hline $\mathbf{9}$ & 400 & 176,64 \\
\hline $\mathbf{9}$ & 600 & 207,94 \\
\hline $\mathbf{9}$ & 1000 & 278,04 \\
\hline $\mathbf{1 1}$ & 200 & 171,03 \\
\hline $\mathbf{1 1}$ & 400 & 252,34 \\
\hline $\mathbf{1 1}$ & 600 & 296,29 \\
\hline $\mathbf{1 1}$ & 1000 & 457,94 \\
\hline $\mathbf{1 2}$ & 400 & 267,29 \\
\hline $\mathbf{1 2}$ & 600 & 332,71 \\
\hline $\mathbf{1 2}$ & 1000 & 469,21 \\
\hline $\mathbf{1 2}$ & 2000 & 732,44 \\
\hline
\end{tabular}

$\mathrm{Na}$ tabela VI, apresentam-se as características gerais das estruturas de sustentação adotadas para o sistema em estudo.

TABELA VI

\begin{tabular}{c|c|c}
\multicolumn{3}{c}{ CARACTERÍSTICAS GERAIS DAS ESTRUTURAS } \\
\hline Código & Modelo & $\begin{array}{c}\text { Custo } \\
\text { (US\$) }\end{array}$ \\
\hline $\mathbf{1}$ & CE1------1 & 44,51 \\
\hline $\mathbf{2}$ & CE1A-----1 & 53,15 \\
\hline $\mathbf{3}$ & CE2------1 & 146,87 \\
\hline $\mathbf{4}$ & CE3------1 & 338,93 \\
\hline $\mathbf{5}$ & CE4------1 & 321,43 \\
\hline $\mathbf{6}$ & CE3CE3---1 & 350,86 \\
\hline
\end{tabular}

\begin{tabular}{c|c|c}
\hline $\mathbf{7}$ & CE2CE3DH-1 & 375,65 \\
\hline $\mathbf{8}$ & CE3D-----1 & 423,44 \\
\hline $\mathbf{9}$ & CE2DCE3--1 & 484,78 \\
\hline
\end{tabular}

\section{G. Resultados}

O sistema teste utilizado é adaptado da literatura. Este sistema é constituído por 50 barras de consumo, 16 ramos existentes e 45 ramos potenciais, duas subestações existentes e duas futuras [6], representado na Fig. 5. O vão básico adotado é de 30 metros entre as estruturas de suspensão [17]. A quantidade de espaçadores, assim como o posicionamento destes, também são definidos em [17].

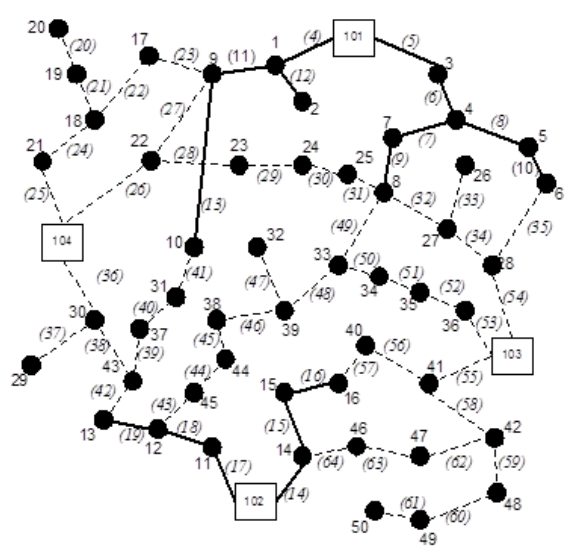

Figura 5. Topologia do sistema teste.

A topologia encontrada para a melhor solução é apresentada na Fig. 6. Verifica-se que algumas barras deste sistema não estão conectadas à subestação porque estas barras não apresentam demanda de potência para o horizonte de planejamento considerado.

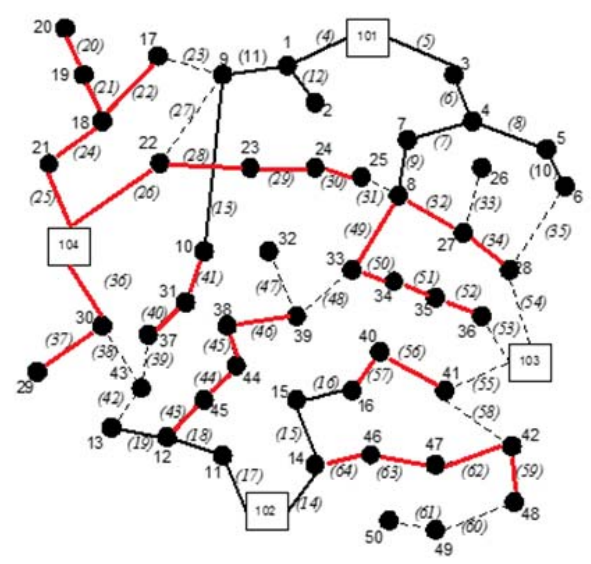

Figura 6. Topologia da melhor solução para o sistema teste.

Na Fig. 7, ilustra-se o comportamento da $F A$ pelo número de iterações do algoritmo BT. A solução inicial apresenta uma $F A$ de alto custo devido às infactibilidades, mas converge em poucas iterações para uma região com $F A s$ de baixo custo, ou seja, sem violações de restrições. 
Função de Adaptação (FA)

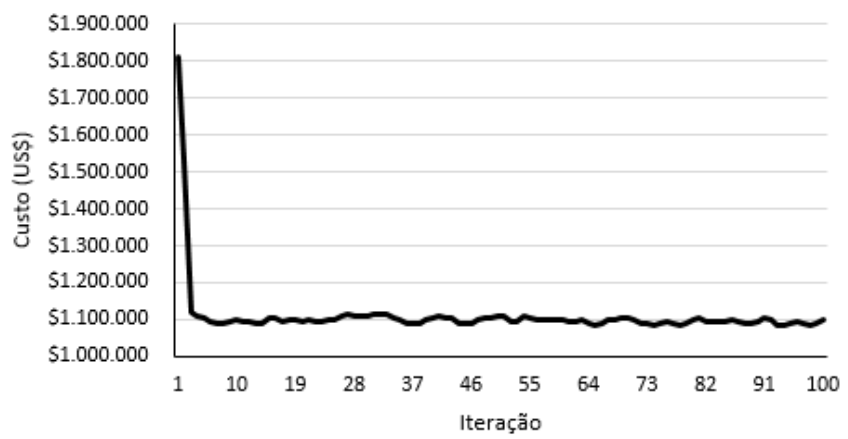

Figura 7. Comportamento da FA para o sistema teste.

$\mathrm{Na}$ tabela VII, apresentam-se os ramos conectados, conforme mostrado na topologia da Fig. 6, e os respectivos tipos (bitola) de cabos.

TABELA VII CARACTERÍSTICAS DOS RAMOS DO SISTEMA

\begin{tabular}{|c|c|c|c|c|c|}
\hline Ramo & $X C_{i}$ & $\begin{array}{c}\text { Modelo } \\
\text { Cabo } \\
\end{array}$ & Ramo & $X C_{i}$ & $\begin{array}{c}\text { Modelo } \\
\text { Caboo } \\
\end{array}$ \\
\hline 4 & 1 & 8 & 35 & 0 & 0 \\
\hline 5 & 1 & 8 & 36 & 1 & 6 \\
\hline 6 & 1 & 8 & 37 & 1 & 1 \\
\hline 7 & 1 & 8 & 38 & 0 & 0 \\
\hline 8 & 1 & 8 & 39 & 0 & 0 \\
\hline 9 & 1 & 8 & 40 & 1 & 1 \\
\hline 10 & 1 & 1 & 41 & 1 & 2 \\
\hline 11 & 1 & 8 & 42 & 0 & 0 \\
\hline 12 & 1 & 5 & 43 & 1 & 2 \\
\hline 13 & 1 & 8 & 44 & 1 & 1 \\
\hline 14 & 1 & 8 & 45 & 0 & 1 \\
\hline 15 & 1 & 8 & 46 & 0 & 1 \\
\hline 16 & 1 & 8 & 47 & 0 & 0 \\
\hline 17 & 1 & 8 & 48 & 1 & 0 \\
\hline 18 & 1 & 8 & 49 & 1 & 8 \\
\hline 19 & 1 & 1 & 50 & 1 & 3 \\
\hline 20 & 1 & 1 & 51 & 1 & 2 \\
\hline 21 & 1 & 1 & 52 & 1 & 8 \\
\hline 22 & 1 & 1 & 53 & 0 & 0 \\
\hline 23 & 0 & 0 & 54 & 0 & 0 \\
\hline 24 & 1 & 4 & 55 & 0 & 0 \\
\hline 25 & 1 & 8 & 56 & 1 & 1 \\
\hline 26 & 1 & 6 & 57 & 1 & 1 \\
\hline 27 & 0 & 0 & 58 & 0 & 0 \\
\hline 28 & 1 & 5 & 59 & 1 & 1 \\
\hline 29 & 1 & 1 & 60 & 0 & 0 \\
\hline 30 & 1 & 1 & 61 & 0 & 0 \\
\hline 31 & 0 & 0 & 62 & 1 & 1 \\
\hline 32 & 1 & 1 & 63 & 1 & 1 \\
\hline 33 & 0 & 0 & 64 & 1 & 1 \\
\hline
\end{tabular}

$\mathbf{3 4} \quad 1$

Na tabela VIII, apresentam-se a altura e o esforço de torção que a estrutura de suspensão estará sujeita $\left(\mathrm{L}_{\mathrm{i}} / \mathrm{F}_{\mathrm{Ri}}\right)$ e, também, o modelo adequado para a estrutura de sustentação para cada barra do sistema. A última coluna representa a resultante do custo associado a cada barra.

TABELA VIII

DEFINIÇÕES DAS ESTRUTURAS MECÂNICAS NAS BARRAS

\begin{tabular}{|c|c|c|c|}
\hline Barra & $\mathbf{L}_{\mathrm{i}} / \mathbf{F}_{\mathrm{Ri}}(\mathrm{m} / \mathrm{DaN})$ & $\begin{array}{c}\text { Modelo de } \\
\text { estrutura }\end{array}$ & Custo (US\$) \\
\hline 1 & $12 / 1000$ & CE2DCE3--1 & 953,99 \\
\hline 2 & $12 / 1000$ & CE3------1 & 808,14 \\
\hline 3 & $12 / 1000$ & CE4------1 & 790,64 \\
\hline 4 & $12 / 400$ & CE2DCE3--1 & 752,07 \\
\hline 5 & $12 / 600$ & CE4------1 & 654,14 \\
\hline 6 & $12 / 400$ & CE3------1 & 606,22 \\
\hline 7 & $12 / 1000$ & CE4------1 & 790,64 \\
\hline 8 & $12 / 400$ & CE2DCE3--1 & 752,07 \\
\hline 9 & $12 / 1000$ & CE4------1 & 790,64 \\
\hline 10 & $12 / 400$ & CE4------1 & 588,72 \\
\hline 11 & $12 / 2000$ & CE4------1 & 1053,87 \\
\hline 12 & $12 / 600$ & CE2DCE3--1 & 817,49 \\
\hline 13 & $12 / 400$ & CE3------1 & 606,22 \\
\hline 14 & $12 / 2000$ & CE2DCE3--1 & $1.217,22$ \\
\hline 15 & $12 / 1000$ & CE4------1 & 790,64 \\
\hline 16 & $12 / 600$ & CE4------1 & 654,14 \\
\hline 17 & $12 / 400$ & CE3------1 & 606,22 \\
\hline 18 & $12 / 600$ & CE2DCE3-1 & 817,49 \\
\hline 19 & $12 / 400$ & CE4------1 & 588,72 \\
\hline 20 & $12 / 400$ & CE3----1 & 606,22 \\
\hline 21 & $12 / 1000$ & CE4------1 & 790,64 \\
\hline 22 & $12 / 600$ & CE4------1 & 654,14 \\
\hline 23 & $12 / 400$ & CE4------1 & 588,72 \\
\hline 24 & $12 / 400$ & CE4------1 & 588,72 \\
\hline 25 & $12 / 400$ & CE3-----1 & 606,22 \\
\hline 26 & -- & -- & -- \\
\hline 27 & $12 / 600$ & CE4------1 & 654,14 \\
\hline 28 & $12 / 400$ & CE3------1 & 606,22 \\
\hline 29 & $12 / 400$ & CE3------1 & 606,22 \\
\hline 30 & 12/1000 & CE4-----1 & 790,64 \\
\hline 31 & $12 / 400$ & CE4------1 & 588,72 \\
\hline 32 & -- & -- & -- \\
\hline 33 & $12 / 1000$ & CE4------1 & 790,64 \\
\hline 34 & $12 / 400$ & CE4------1 & 588,72 \\
\hline 35 & $12 / 400$ & CE4----1 & 588,72 \\
\hline 36 & $12 / 1000$ & CE3------1 & 808,14 \\
\hline 37 & $12 / 400$ & CE4-----1 & 588,72 \\
\hline
\end{tabular}




\begin{tabular}{c|c|c|c}
\hline $\mathbf{3 8}$ & $12 / 600$ & CE4------1 & 654,14 \\
\hline $\mathbf{3 9}$ & $12 / 400$ & CE3------1 & 606,22 \\
\hline $\mathbf{4 0}$ & $12 / 600$ & CE4------1 & 654,14 \\
\hline $\mathbf{4 1}$ & $12 / 400$ & CE3------1 & 606,22 \\
\hline $\mathbf{4 2}$ & $12 / 600$ & CE4------1 & 654,14 \\
\hline $\mathbf{4 3}$ & --- & -- & -- \\
\hline $\mathbf{4 4}$ & $12 / 400$ & CE4-----1 & 588,72 \\
\hline $\mathbf{4 5}$ & $12 / 400$ & CE4------1 & 588,72 \\
\hline $\mathbf{4 6}$ & $12 / 400$ & CE4------1 & 588,72 \\
\hline $\mathbf{4 7}$ & $12 / 400$ & CE4------1 & 588,72 \\
\hline $\mathbf{4 8}$ & $12 / 400$ & CE3------1 & 606,22 \\
\hline $\mathbf{4 9}$ & -- & --- & -- \\
\hline $\mathbf{5 0}$ & -- & -- & -- \\
\hline Total & & & $31.220,79$ \\
\hline
\end{tabular}

-

$\mathrm{Na}$ tabela IX, definem-se as estruturas de suspensão e sustentação que são utilizadas entre os ramos que conectam as barras do sistema, de acordo com as normas propostas em [17]. Apresentam-se nas duas últimas colunas os custos associados a estas estruturas.

TABELA IX

DEFINIÇÕES DAS ESTRUTURAS MECÂNICAS NOS RAMOS

\begin{tabular}{c|c|c|c|c|c|c}
\hline $\mathbf{R a m o}$ & $\begin{array}{c}\mathbf{L}_{\mathbf{i}} / \mathbf{F}_{\mathbf{R i}} \\
(\mathbf{m} / \mathbf{D a N})\end{array}$ & $\begin{array}{c}\mathbf{C E 4} \\
\mathbf{1}\end{array}$ & $\begin{array}{c}\mathbf{C E 1 A -} \\
\mathbf{1}\end{array}$ & $\begin{array}{c}\text { CE1- } \\
\mathbf{1}\end{array}$ & $\begin{array}{c}\text { Custo } \\
\text { (US\$) }\end{array}$ & $\begin{array}{c}\text { Espaçador } \\
\text { (US\$) }\end{array}$ \\
\hline $\mathbf{4}$ & $11 / 200$ & 5 & 6 & 67 & $18.248,56$ & $2.110,68$ \\
\hline $\mathbf{5}$ & $11 / 200$ & 4 & 4 & 52 & $14.074,64$ & $1.623,60$ \\
\hline $\mathbf{6}$ & $11 / 200$ & 6 & 6 & 75 & $20.465,34$ & $2.354,22$ \\
\hline $\mathbf{7}$ & $11 / 200$ & 4 & 5 & 60 & $16.023,14$ & $1.867,14$ \\
\hline $\mathbf{8}$ & $11 / 200$ & 6 & 6 & 75 & $20.465,34$ & $2.354,22$ \\
\hline $\mathbf{9}$ & $11 / 200$ & 6 & 6 & 75 & $20.465,34$ & $2.354,22$ \\
\hline $\mathbf{1 0}$ & $11 / 200$ & 4 & 5 & 60 & $16.023,14$ & $1.867,14$ \\
\hline $\mathbf{1 1}$ & $11 / 200$ & 6 & 7 & 83 & $22.413,84$ & $2.597,76$ \\
\hline $\mathbf{1 2}$ & $11 / 200$ & 6 & 6 & 75 & $20.465,34$ & $2.354,22$ \\
\hline $\mathbf{1 3}$ & $11 / 200$ & 14 & 14 & 175 & $47.752,46$ & $5.493,18$ \\
\hline $\mathbf{1 4}$ & $11 / 200$ & 7 & 7 & 91 & $24.630,62$ & $2.841,30$ \\
\hline $\mathbf{1 5}$ & $11 / 200$ & 7 & 7 & 91 & $24.630,62$ & $2.841,30$ \\
\hline $\mathbf{1 6}$ & $11 / 200$ & 5 & 6 & 67 & $18.248,56$ & $2.110,68$ \\
\hline $\mathbf{1 7}$ & $11 / 200$ & 5 & 6 & 67 & $18.248,56$ & $2.110,68$ \\
\hline $\mathbf{1 8}$ & $11 / 200$ & 6 & 6 & 75 & $20.465,34$ & $2.354,22$ \\
\hline $\mathbf{1 9}$ & $11 / 200$ & 8 & 9 & 105 & $28.589,00$ & $3.301,32$ \\
\hline $\mathbf{2 0}$ & $11 / 200$ & 6 & 6 & 75 & $20.465,34$ & $2.354,22$ \\
\hline $\mathbf{2 1}$ & $11 / 200$ & 4 & 5 & 60 & $16.023,14$ & $1.867,14$ \\
\hline $\mathbf{2 2}$ & $11 / 200$ & 8 & 8 & 97 & $26.640,50$ & $3.057,78$ \\
\hline $\mathbf{2 3}$ & -- & -- & -- & -- & -- & -- \\
\hline $\mathbf{2 4}$ & $11 / 200$ & 6 & 6 & 75 & $20.465,34$ & $2.354,22$ \\
\hline $\mathbf{2 5}$ & $11 / 200$ & 4 & 5 & 60 & $16.023,14$ & $1.867,14$ \\
\hline $\mathbf{2 6}$ & $11 / 200$ & 7 & 7 & 91 & $24.630,62$ & $2.841,30$ \\
\hline
\end{tabular}

\begin{tabular}{|c|c|c|c|c|c|c|}
\hline 27 & -- & -- & -- & -- & -- & -- \\
\hline 28 & $11 / 200$ & 6 & 7 & 83 & $22.413,84$ & $2.597,76$ \\
\hline 29 & $11 / 200$ & 5 & 6 & 67 & $18.248,56$ & $2.110,68$ \\
\hline 30 & $11 / 200$ & 4 & 4 & 52 & $14.074,64$ & $1.623,60$ \\
\hline 31 & -- & -- & -- & -- & -- & -- \\
\hline 32 & $11 / 200$ & 7 & 7 & 91 & $24.630,62$ & $2.841,30$ \\
\hline 33 & -- & -- & -- & -- & -- & -- \\
\hline 34 & $11 / 200$ & 6 & 6 & 75 & $20.465,34$ & $2.354,22$ \\
\hline 35 & -- & -- & -- & -- & -- & -- \\
\hline 36 & $11 / 200$ & 5 & 6 & 67 & $18.248,56$ & $2.110,68$ \\
\hline 37 & $11 / 200$ & 6 & 6 & 75 & $20.465,34$ & $2.354,22$ \\
\hline 38 & -- & -- & -- & -- & -- & -- \\
\hline 39 & -- & -- & -- & -- & -- & -- \\
\hline 40 & $11 / 200$ & 3 & 4 & 44 & $11.857,86$ & $1.380,06$ \\
\hline 41 & $11 / 200$ & 6 & 6 & 75 & $20.465,34$ & $2.354,22$ \\
\hline 42 & -- & -- & -- & -- & -- & -- \\
\hline 43 & $11 / 200$ & 4 & 5 & 60 & $16.023,14$ & $1.867,14$ \\
\hline 44 & $11 / 200$ & 4 & 4 & 52 & $14.074,64$ & $1.623,60$ \\
\hline 45 & $11 / 200$ & 6 & 6 & 75 & $20.465,34$ & $2.354,22$ \\
\hline 46 & $11 / 200$ & 6 & 7 & 83 & $22.413,84$ & $2.597,76$ \\
\hline 47 & -- & -- & -- & -- & -- & -- \\
\hline 48 & -- & -- & -- & -- & -- & -- \\
\hline 49 & $11 / 200$ & 9 & 9 & 113 & $30.805,78$ & $3.544,86$ \\
\hline 50 & $11 / 200$ & 3 & 4 & 44 & $11.857,86$ & $1.380,06$ \\
\hline 51 & $11 / 200$ & 4 & 4 & 52 & $14.074,64$ & $1.623,60$ \\
\hline 52 & $11 / 200$ & 4 & 4 & 52 & $14.074,64$ & $1.623,60$ \\
\hline 53 & -- & -- & -- & -- & -- & -- \\
\hline 54 & -- & -- & -- & -- & -- & -- \\
\hline 55 & -- & -- & -- & -- & -- & -- \\
\hline 56 & $11 / 200$ & 4 & 5 & 60 & $16.023,14$ & $1.867,14$ \\
\hline 57 & $11 / 200$ & 4 & 5 & 60 & $16.023,14$ & $1.867,14$ \\
\hline 58 & -- & -- & -- & -- & -- & -- \\
\hline 59 & $11 / 200$ & 4 & 5 & 60 & $16.023,14$ & $1.867,14$ \\
\hline 60 & -- & -- & -- & -- & -- & -- \\
\hline 61 & -- & -- & -- & -- & -- & -- \\
\hline 62 & $11 / 200$ & 6 & 6 & 75 & $20.465,34$ & $2.354,22$ \\
\hline 63 & $11 / 200$ & 6 & 6 & 75 & $20.465,34$ & $2.354,22$ \\
\hline 64 & $11 / 200$ & 6 & 7 & 83 & $22.413,84$ & $2.597,76$ \\
\hline $\begin{array}{c}\text { TOTA } \\
\text { L }\end{array}$ & & & & & $901.531,80$ & $104.126,90$ \\
\hline
\end{tabular}

Na tabela X, comparam-se os resultados da solução da configuração inicial com a melhor solução encontrada pelo método proposto. Tem-se que a redução do custo para o planejamento corresponde a $76,96 \%$ e para o projeto corresponde a $38,62 \%$, totalizando uma redução geral dos custos de $41,17 \%$. 
TABELA X

CUSTOS GERAIS PARA O SISTEMA II

\begin{tabular}{c|c|c|c}
\hline $\begin{array}{c}\text { Descrição dos } \\
\text { Custos }\end{array}$ & $\begin{array}{c}\text { Configuração } \\
\text { Inicial (US\$) }\end{array}$ & $\begin{array}{c}\text { Melhor } \\
\text { Solução (US\$) }\end{array}$ & $\begin{array}{c}\text { Redução } \\
\mathbf{( \% )}\end{array}$ \\
\hline Planejamento & $120.335,92$ & $27.728,32$ & 76,96 \\
\hline Projeto & $1.689 .346,79$ & $1.036 .879,49$ & 38,62 \\
\hline $\begin{array}{c}\text { Total da } \\
\text { solução }\end{array}$ & $1.809 .682,71$ & $1.064 .607,81$ & 41,17 \\
\hline
\end{tabular}

\section{H. Discussão dos Resultados}

Os resultados obtidos com testes neste sistema mostram que a melhor solução encontrada pelo método proposto reduziu o custo total do problema em $41,17 \%$ quando comparado com os custos obtidos pela solução da configuração inicial.

A metodologia proposta atendeu às restrições de qualidade impostas à rede de distribuição, garantindo a alimentação de todas as barras de demanda. O processo de intensificação demonstrou ser importante para garantir o atendimento das restrições, pois a troca de condutores possibilitou o cumprimento das equações (3), (4) e (5), e reduziu as perdas geradas na linha (equação (6)).

Os resultados demonstram também que o custo dos projetos das estruturas mecânicas é dominante ao custo de planejamento do sistema elétrico, tornando pertinente para as concessionárias de energia considerar ambos para a tomada de decisão de construção e de modernização das redes de distribuição.

\section{V.CONCLUSÕES}

O problema de planejamento e projetos de sistemas de distribuição de energia elétrica é formulado como um problema de programação não linear inteiro misto (PNLIM) em que foi proposto para solução deste uma metodologia baseada na técnica heurística de Busca Tabu (BT), a fim de minimizar os custos, garantindo o cumprimento das restrições físicas, econômicas e operacionais do sistema, sendo, assim, possível de ser implementada em circuitos reais pelas concessionárias de energia elétrica. A metodologia contemplou a solução de qualquer sistema genérico, sendo testada para um sistema com 50 barras e quatro subestações.

A metodologia proposta em $B T$ mostrou-se robusta, em que a melhor solução apresentou redução significativa dos custos, comparando-se com a solução inicial. A consideração do projeto dos sistemas mecânicos se mostrou fundamental para o problema de planejamento e projetos de sistemas de distribuição de energia elétrica, pois os custos associados aos projetos refletem substancialmente nos custos gerais do sistema, evidenciando a importância do modelo proposto neste trabalho.

\section{AGRADECIMENTOS}

Os autores agradecem a revisão deste manuscrito ao Prof. Alberto Aparecido da Cunha.

\section{REFERÊNCIAS}

[1] T. Gönen, "Electric Power Distribution System Engineering", McGraw - Hill Series in Electrical Engineering, 1986.

[2] A. M. Cossi, "Planejamento de redes de distribuição de energia elétrica de média e baixa tensão". Tese de Doutorado, Faculdade de Engenharia de Ilha Solteira - UNESP, 2008.
[3] G.L.Thompson e D.L. Wall. "A Branch and Bound Model for Choosing Optimal Substation Locations", IEEE Transactions on Power Apparatus and Systems, Vol. PAS-100, No. 5, pp. 2683-2688, 1981.

[4] D.I. Sun; D.R. Farris; P.J. Cote; R.R. Shoults and M.S. Chen "Optimal distribution substation and primary feeder via the fixed charge network formulation", IEEE Transactions on Power Apparatus and Systems, New York, v.101, n.3, p.602-609, 1982.

[5] S. K. Goswami, "Distribution System Planning Using Branch Exchange Technique", IEEE Transactions on Power Systems, USA, 12(2): 718723. 1997.

[6] J. L. Bernal Agustin, I. J. Ramirez Rosado, "Genetic algorithm applied to the design of large power distribution systems," IEEE Transactions on Power Systems, vol. 13, no. 2, pp. 696-703, 1998.

[7] I.J. Ramirez-Rosado, J.L. Bernal-Augustin, "Reliability and Costs Optimization for Distribution Networks Expansion Using and Evolutionary Algorithm", IEEE Transactions on Power Systems, Vol. 16, No.1, pp. 111-118.

[8] E. C. Yeh, S. S. Venkata, and Z. Sumic, "Improved distribution system planning using computational evolution," IEEE Transactions on Power Systems, vol. 11, pp. 668-674, 1996.

[9] F. A. B. Lucero, "Planejamento De Sistemas De Distribuição De Energia Elétrica Utilizando Algoritmo Busca Tabu". Tese de Mestrado, Faculdade de Engenharia de Ilha Solteira - UNESP, 2003.

[10] J.F. Gomez, H.M. Khodr, P.M. Oliveira; L. Ocque; J.M. Yusta; R. Villasana; A.J. Urdaneta "Ant colony system algorithm for the planning of primary distribution circuits", IEEE Transactions on Power Systems, New York, v.19, n.2, p.996-1004, 2004.

[11] I.J. Ramirez-Rosado, J.L. Bernal-Augustin, "Reliability and Costs Optimization for Distribution Networks Expansion Using and Evolutionary Algorithm", IEEE Transactions on Power Systems, Vol. 16, No.1, pp. 111-118.

[12] I.J. Ramirez-Rosado, J.A. Domínguez-Navarro, "Possibilist Model Based on Fuzzy Sets for the Multiobjective Optimal Planning of Power Electric Distribution Networks", IEEE Transaction on Power System, Vol. 19, pp. 1801-1810, 2004.

[13] B. R. Pereira Junior, A. M. Cossi, J. Contreras and J. R. S. Mantovani, "Multiobjective multistage distribution system planning using tabu search", IET Generation, Transmission \& Distribution, v. 8, Iss. 1, p.3545, 2014.

[14] A. Cossi, L. W. d. Silva, J. Mantovani and R. Romero, "Primary Power Distribution Systems Planning Taking Into Account Reliability, Operation and Expansion Costs", IET Generation, Transmission \& Distribution, v. 6, Iss. 3, p.172-180, 2012

[15] S. G. Benetti de Padua, A. M. Cossi , J. R. S. Mantovani and, "Planning Medium-Voltage Electric Power Distribution Systems through a Scatter Search Algorithm," in IEEE Latin America Transactions, vol. 13, no. 8, pp. 2637-2645, Aug. 2015.

[16] Agência Nacional de Energia Elétrica - ANEEL, "Procedimentos de Distribuição de Energia Elétrica no Sistema Elétrico Nacional PRODIST - Módulo 8 - Qualidade da Energia Elétrica". Revisão 6, 2015.

[17] Companhia Paulista de Força e Luz, "Projeto de Rede de Distribuição Cálculo Mecânico". 2011.

[18] F. Glover, "*Tabu Search Fundamentals and Uses*", University of Colorado, Boulder, Colorado, April 1995.

[19] C. S. Cheng; D. A. Shirmohammadi. "Three-phase power flow method for real-time distribution system analysis". IEEE Transactions on Power Systems, New York, v.10, n.2, p.671-679, 1995.

[20] Companhia Paulista de Força e Luz, "Rede Primária Compacta $15 \mathrm{kV}$ e $25 \mathrm{kV}$ - Estruturas Básicas - Montagem". 2011.

[21] F. Mendoza, J.L. Bernal-Agustin, J.A. Dominguez-Navarro, "NSGA and SPEA applied to multiobjective design of power distribution systems" IEEE Transactions on Power Systems, New York, v.21, n.4, pp.19381945,2006

Vinicius Carnelossi da Cunha nasceu em Catanduva, São Paulo, Brasil. Graduado em Engenharia Elétrica pela Universidade Estadual Paulista (UNESP), Campus de Ilha Solteira, São Paulo, Brasil, em 2013. Atualmente está desenvolvendo atividades acadêmicas para obter o grau de Mestre em Engenharia Elétrica pela Universidade Estadual de Campinas (UNICAMP). 
José Roberto Sanches Mantovani é graduado em Engenharia Elétrica pela Universidade Estadual Paulista (UNESP), Campus de Ilha Solteira, São Paulo, Brasil, em 1981. Pós-Graduado em Engenharia Elétrica em nível de mestrado e doutorado pela Universidade Estadual de Campinas (UNICAMP), São Paulo, Brasil, 1987 e 1995. Atualmente é professor titular do Departamento de Engenharia Elétrica (DEE) e membro do Laboratório de Planejamento de Sistemas de Energia Elétrica (LaPSEE) na UNESP. 